\title{
Variant Alzheimer Disease with Spastic Paraparesis: A Rare Presenilin-1 Mutation
}

\author{
Jacqueline A. Pettersen, David G. Patry, Peter H. St. George-Hyslop, Bernadette Curry
}

Can. J. Neurol. Sci. 2011; 38: 659-661

Variant Alzheimer's with spastic paraparesis (varAD) is a rare clinical entity characterized by early-onset dementia and gait disorder with lower extremity spasticity and weakness. ${ }^{1-4}$ There is some phenotypic variability in that either the dementia or paraparesis may present first or in isolation and myoclonus, parkinsonian and psychiatric features may be present. ${ }^{1-3}$ Pathologically, the most striking features are $\beta$-amyloid-positive "cotton wool" plaques and degeneration of corticospinal tracts. ${ }^{1}$ Typical Alzheimer pathology is commonly observed and amyloid angiopathy may be present., ${ }^{2,3}$ Genetic testing typically reveals a chromosome 14 presenilin-1 mutation (PSEN1) involving exon $9^{1}$, resulting in increased production of $\mathrm{A} \beta 42$, a major component of amyloid plaques in Alzheimer's disease (AD).

We describe a case of varAD in which the PSEN1 missense mutation involved substitution of leucine for proline at codon 264. This specific mutation has been associated with familial AD but has only been described in one other family with varAD. ${ }^{4}$

\section{Case Report}

\section{Clinical Description}

A 57-year-old woman died following a progressive course of myoclonus, global cognitive and behavioural decline, and spastic paraparesis. The myoclonus began approximately 15 years before death and involved both arms but in the final year, became stimulus-responsive and more diffuse, involving the legs and trunk. During the final two years of life, she developed stooped posture with marked instability and truncal rigidity, which were unresponsive to levodopa. Cognitive and behavioural changes were insidious and first noted five years before death with a decline in hygiene and social interaction. Within two years, she stopped driving following minor motor vehicle accidents and episodes of getting lost. Concurrently, she experienced difficulties with calculations and banking. Gait problems began within the last two years of life, resulting in frequent falls and eventually, confinement to a wheelchair. Physical examination revealed leg spasticity and hyperreflexia with a "shuffling" and unsteady gait.

She was brought to medical attention two years prior to death when she was admitted to the psychiatry service for behavioural decompensation (confusion, poor self care, vague auditory hallucinations). A modified mini-mental status exam (MMSE) was scored at 18/30 while formal neuropsychological testing revealed diffuse cognitive impairment with exceptional difficulties on spatial/constructional tasks and retrieval for both visual and auditory information (recognition was comparatively preserved). She was discharged to a special care home and followed regularly through the cognitive assessment clinic. Over the subsequent two years, she was noted to have frontal release signs, ideomotor apraxia, significant word-finding difficulties, markedly slowed responses, and emotional lability. A repeat MMSE one year prior to death was $9 / 30$.

Brain magnetic resonance imaging (MRI) revealed multiple areas of T2-weighted white matter hyperintensities in periventricular and subcortical regions bilaterally. These changes were thought to represent small vessel ischemic disease although she had no vascular risk factors. An electroencephalogram, SPECT scan, cerebrospinal fluid studies, abdominal ultrasound, and blood work for TSH, B 12 , VDRL, lactate and Huntington's disease, were non-diagnostic. The patient and her family had consented to autopsy. While the details were limited, there was a known family history of dementia and "gait problems" affecting her mother, maternal uncle, and two maternal aunts.

\section{Neuropathology}

Gross

The brain was mildly atrophic (weight: $1358 \mathrm{~g}$ ), most pronounced posteriorly with concomitantly enlarged ventricular posterior horns. The posterior parietal and occipital lobe white matter was reduced and abnormal in colour with a "grayish" appearance. While hippocampal atrophy was minimal, the fornix was reduced in size. The substantia nigra and locus ceruleus were significantly less pigmented than normal and this was confirmed on microscopic sections.

\section{Microscopic}

The most striking abnormality was the presence of numerous "cotton wool" plaques in the superficial layers of all cerebral lobes, especially the frontal and parietal lobes, as well as the

\footnotetext{
From the Department of Medicine (Neurology) (JAP), University of British Columbia, Vancouver; Northern Medical Program (JAP), University of Northern British Columbia, Prince George, BC; Departments of Clinical Neurosciences (DGP, BC) and Pathology (BC), University of Calgary, Calgary, AB; Tanz Centre for Research in Neurodegenerative Diseases (PHSGH), Department of Medicine, University of Toronto, ON, Canada; Cambridge Institute for Medical Research (PHSGH),

Department of Clinical Neurosciences, University of Cambridge, Cambridge, UK. Received February 17, 2011. Final Revisions Submitted March 3, 2011. Correspondence to: Jacqueline Pettersen, Northern Medical Program, 3333 University Way, University of Northern British Columbia, Prince George, British Columbia, V2N 4Z9, Canada.
} 

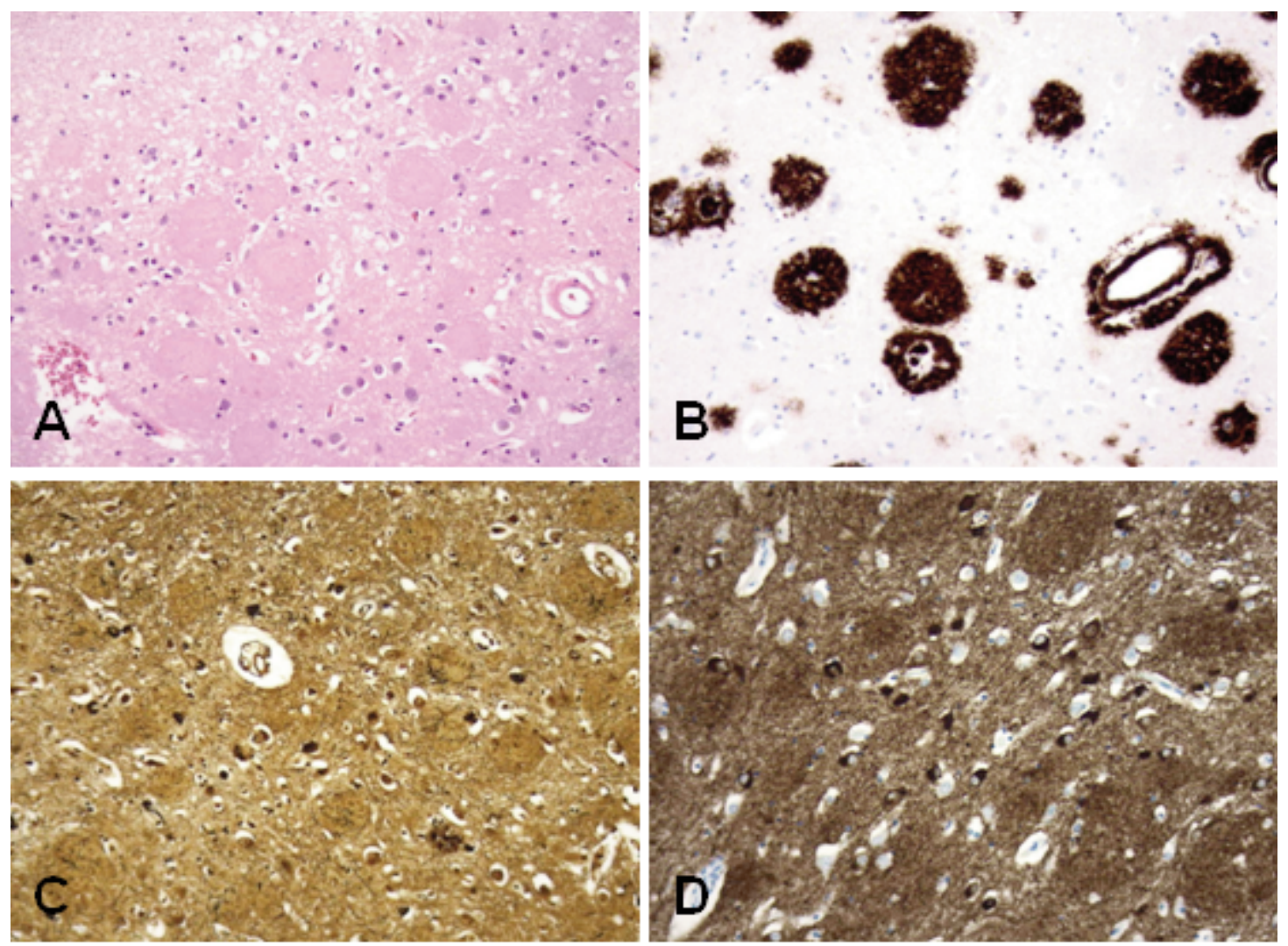

Figure: "Cotton wool" plaques from the frontal lobe as seen at $20 \mathrm{X}$ magnification on sections stained with A) hematoxylin and eosin; B) $\beta$-amyloid immunostain; C) Bielschowsky silver stain; and D) Tau stain. Note the distinct borders, strong positivity to $\beta$-amyloid but only minor neuritic pathology within these plaques, differentiating them from neuritic plaques.

basal ganglia, limbic areas, cerebellum, brainstem and lumbar spinal cord. These plaques were visible in hematoxylin and eosin-stained sections as large, round homogeneous eosinophilic structures with distinct borders, lacking a central core, (Figure 1A) and were immunoreactive to beta-amyloid (Figure 1B) but lacked congophilia. While numerous intraneuronal neurofibrillary tangles were present in the surrounding parenchyma, as revealed by Bielschowsky-stained sections, there was only minor neuritic pathology within the "cotton wool" plaques (Figure 1C). Tau stain revealed numerous dense threads and tau-positive neurons (Figure 1D). Typical neuritic plaques were also evident in these same brain regions and there were focal areas of neuronal loss associated with spongiosis in the frontal, parietal and temporal lobes. Within the primary motor cortex, Betz cells were scant and atrophic, with relative sparing of layer three. Blood vessels within cortical and subcortical regions and the anterior spinal artery appeared thick-walled and stiff and reacted positively to congo red staining, consistent with amyloid angiopathy. Three small infarcts with neuronal loss and gliosis were identified: two within the occipital lobe sparing the primary visual cortex and the third in the neocortex inferior to the amygdala. Areas of white matter pallor in the frontal and occipital lobes were also suggestive of ischemia. Spinal cord sections stained with luxol fast blue revealed loss of myelinated fibers within the lateral and ventral corticospinal tracts.

\section{Genetic Testing}

Genetic sequencing of the entire open reading frame of the PSEN1 gene (obtained from frozen brain tissue) revealed a point mutation in exon 8 ( $\mathrm{C}>\mathrm{T}$ at nucleotide position 1039) causing an amino acid change Pro264Leu (CCG>CTG).

\section{Clinical-Pathological-Genetic Correlation}

This clinical presentation correlates well with the distribution of neuropathological findings. Plaques, neurofibrillary tangles, neuronal threads, and neuronal loss would account for the cognitive and behavioural decline, while changes in the substantia nigra and locus ceruleus correlate with parkinsonian features. Presence of congophilic angiopathy in association with multiple infarcts and more diffuse ischemic change accounts for the T2-weighted hyperintensities on MRI and correlates with the white matter loss in the posterior parietal and occipital lobes. Corticospinal tract degeneration and atrophy, along with presence of cotton wool plaques and loss of Betz cells in the primary motor cortex, corresponds with the progressive gait difficulties and upper motor neuron signs in the legs. The genetic mutation in exon 8 (P264L) has been described in the context of varAD in at least one other case to date ${ }^{4}$, and therefore, further supports our diagnosis. 


\section{Discussion}

The pattern of clinical and neuropathological findings is consistent with a diagnosis of varAD. The clinical findings of behavioural change, parkinsonian features, and myoclonus have been reported in varAD previously, ${ }^{1,2}$ while white matter changes secondary to amyloid angiopathy have also been described in some kinships ${ }^{3}$. The PSEN1 mutation determined in the current study, although not the most common associated genotype, would be consistent with a diagnosis of varAD ${ }^{4}$. Similar to the one previous report of an exon 8 P264L mutation in varAD, and in contrast to typical $\mathrm{AD}$, our case presented more with visuospatial deficits and concomitant posterior cerebral atrophy, with relative preservation of recognition memory and associated hippocampal volume. ${ }^{4}$

Variant Alzheimer's with spastic paraparesis has been linked to several mutations in the PSEN1 gene on chromosome 14. Almost two decades ago, a locus for hereditary spastic paraparesis was mapped to chromosome 14, however, it was not until more recently that that the co-presentation of spastic paraparesis and $\mathrm{AD}$ was described in association with PSEN1 mutations. Interestingly, cotton wool plaques are invariably present when both of these features occur. ${ }^{1-5}$ Deletions of exon 9 are most frequently reported, however, several other mutations including those involving exon 4 (L85P), exon 5 (Y154N) and exon 8 have also been described (for further details, please refer to the following website:http://www.molgen.ua.ac.be/AD Mutations/default.cfm?MT $=0 \& \mathrm{ML}=0 \&$ Page $=$ Home) Notably, exon 8 mutations ${ }^{3}$, including those involving $\mathrm{P} 264 \mathrm{~L}^{4}$ seem to be associated with more pronounced white matter abnormalities likely secondary to amyloid angiopathy ${ }^{3}$, as was evident in our case.

The current case represents the second report of VarAD due to exon $8 \mathrm{P} 264 \mathrm{~L}^{4}$, which is a mutation more typically associated with $\mathrm{AD}$ in the absence of spastic paraparesis. That this and other PSEN1 mutations, such as E280Q (exon 8), can occur with either classic $\mathrm{AD}$ or $\operatorname{varAD}^{5}$ supports the hypothesis that a genetic (or environmental) modifier must also be present ${ }^{5}$ that is yet to be elucidated. Interestingly, while there have been at least 30 PSEN1 exon 8 mutations described to date that account for about $17 \%$ of all PSEN1 mutations, (http://www.molgen. ua.ac.be/ADMutations/default.cfm?MT $=0 \& M L=0 \&$ Page $=$ Hom e) only three have been implicated in VarAD. How the phenotypic variations of typical $A D$ versus varAD are mediated from the same genotype is unclear and necessitates further study.

In summary, varAD is a rare, but clinically distinct syndrome that can be confirmed on pathological and genetic analyses. It should be considered in the differential diagnosis of familial early-onset dementia and/or spastic paraparesis. Further, the specific genetic mutation exon 8 P264L may manifest in, not only a typical AD syndrome, but also, as in our case, an atypical $\mathrm{AD}$ with more posterior cortical involvement, pronounced white matter disease, spastic paraparesis and the unusual neuropathological finding of cotton wool plaques.

\section{ACKNOWLEDGEMENTS}

This work was supported by The Canadian Institutes of Health Research (JP, PHSGH), Alberta Health Foundation for Medical Research (JP), Alzheimer Society of Ontario (PHSGH), Howard Hughes Medical Institute (PHSGH), and the Wellcome Trust (PHSGH).

\section{REFERENCES}

1. Smith MJ, Kwok JBJ, McLean CA, et al. Variable phenotype of Alzheimer's disease with spastic paraparesis. Ann Neurol. 2001;49:125-9.

2. Tabira T, Chui DH, Nakayama H, Kuroda S, Shibuya M. Alzheimer's disease with spastic paraparesis and cotton wool type plaques. J Neurosci Res. 2002;70:367-72.

3. O'Riordan S, McMonagle P, Janssen JC, et al. Presenilin-1 mutation (E280G), spastic paraparesis, and cranial white-matter abnormalities. Neurology. 2002;59:1108-10.

4. Jacquemont M-L, Campion D, Hahn V, et al. Spastic paraparesis and atypical dementia caused by PSEN1 mutation (P264L), responsible for Alzheimer's disease. J Med Genet. 2002;39:e2.

5. Rogaeva E, Bergeron C, Sato C, et al. PS1 Alzheimer's disease family with spastic paraparesis: The search for a gene modifier. Neurology. 2003;61:1005-7. 